\title{
ULTRASONIC BENCHMARKING WITH UTDefect
}

\author{
Per-Åke Jansson and Anders Boström \\ Department of Applied Mechanics, Chalmers University of Technology, \\ SE-412 96 Göteborg, Sweden
}

\begin{abstract}
UTDefect is a program for simulation of ultrasonic testing with emphasis on applications within the nuclear power industry. The entire testing process, including the ultrasonic transmitter, the receiver, and scattering from various types of defects of simple shape, is modelled. The basic idea behind UTDefect is to use solutions to the elastodynamic wave equation that are esentially exact. For the 2009 benchmark problems the results obtained from UTDefect are in most cases in fairly good agreement with the experimental data from CEA.
\end{abstract}

Keywords: Ultrasound, Calibration, Side-Drilled Hole, Flat-bottomed Hole, Benchmark, Cladding PACS: 43.20.Fn, 43.35.Zc, 46.40.Cd.

\section{INTRODUCTION}

Mathematical modelling and simulation of ultrasonic testing are useful in a number of ways. It helps in the physical interpretation and understanding of test results and it is easy to perform parametric studies, e.g. to develop testing procedures or investigate worst cases.

All models need to be verified by comparisons with experiments and/or other models. For this purpose the World Federation of NDE Centers has proposed benchmark problems for ultrasonic testing during a couple of years. In the 2009 problems three side-drilled holes, one flat-bottomed hole, and 8 rectangular back-wall breaking cracks were investigated by two pulse-echo contact probes. The experiments were carried out by the Commissariat à l'énergie atomique (CEA) in France. The purpose is to compare these experiments with the program UTDefect and also to report recent advances in the program.

\section{THE PROGRAM UTDefect}

The computer program UTDefect has been developed for almost two decades at Chalmers University of Technology with the aim to model typical applications within the nuclear power industry. The development has been reported in a large number of papers, conference proceedings, technical reports, and theses.

The main idea behind UTDefect is to use analytical or semi-analytical methods as much as possible for the scattering by defects. This gives methods of high accuracy that do not employ any approximations (like ray or Kirchhoff approximations) but greatly limit 
the shape of the defects. Thus simple shapes like a side-drilled hole and a spherical void or inhomogeneity are treated by separation-of-variables. Strip-like, circular, and rectangular cracks are treated by hypersingular integral equation methods, which may be regarded as semi-analytical due to the global expansions of the crack-opening displacements that are used (in contrast to methods like BEM or FEM where local expansions over an element are used).

The type of methods used also restricts the material of the tested component to be homogeneous and isotropic, although anisotropic components are possible for strip-like and rectangular cracks. Material attenuation is included as viscous losses. The component is also assumed to be thick-walled, so no multiple scattering between the scanning surface and the defect is taken care of. However, most defect types may be located close to a back-wall of the component, and the strip-like crack can also be surface-breaking.

UTDefect models the whole testing situation in that, except for the scattering by the defect, models of the ultrasonic probes used in transmission and reception are included, as is the calibration by a side-drilled hole, flat-bottomed hole, or a back-wall reflection. The transmitting probe can be of any type and frequency, and it can be rectangular or elliptical. It may be planar or focussed and of contact or immersion type. The model of the probe specifies the traction it exerts on the scanning surface and the field from the probe is straightforwardly calculated by Fourier transform methods. The action of the receiving probe is given by a reciprocity argument.

UTDefect basically works in the frequency domain. By a Fourier transform it is possible to obtain time domain results. Thus standard A, B, C scans are obtained as output. For a $\mathrm{C}$ scan it is often enough to use a single frequency.

The development of UTDefect is on-going. Strip-like and rectangular cracks in an anisotropic cladding with a corrugated interface to the base material is just being included. Work is in progress to include a non-planar back-wall using BEM coupled to hypersingular integral equations for cracks.

\section{THE 2009 ULTRASONIC BENCHMARK PROBLEMS}

In the 2009 ultrasonic benchmark problems experimental tests were performed on a planar block containing rectangular surface-breaking cracks of different heights and extensions. Tests were also made on side-drilled holes and flat-bottomed holes. The experimental data were provided by CEA in France.

The test block was made of stainless steel with a density of $7950 \mathrm{~kg} / \mathrm{m}^{3}$. The wave speeds for longitudinal and transverse waves were $5750 \mathrm{~m} / \mathrm{s}$ and $3150 \mathrm{~m} / \mathrm{s}$, respectively. Two different types of contact probes with different apertures were used, both radiating $45^{\circ}$ transverse waves. For one of the probes, in the following referred to as WB45, waves were generated by a rectangular crystal with dimensions 22 by $20 \mathrm{~mm}$. The center frequency was 2 MHz. For the other probe (MSWQC45) a circular crystal of diameter $6.35 \mathrm{~mm}$ was used with a center frequency of $2.25 \mathrm{MHz}$. A more detailed description of the probes is given at the website of the World Federation of NDE Centers (www.wfndec.org). Tests were also performed for a linear phased array generating longitudinal waves. However, no comparison with these results will be made in this paper.

\section{The Side-Drilled Hole}

Experimental data are given for ultrasonic responses from side-drilled holes of length $60 \mathrm{~mm}$ and different diameters $(1,1.5$ and $2 \mathrm{~mm})$. The centers were positioned at a depth of $30 \mathrm{~mm}$, and the axes were all oriented parallel to the surface of the block. 
TABLE 1. Response from side-drilled holes of different diameters.

\begin{tabular}{|c|c|c|c|c|}
\hline Probe & Diameter $(\mathrm{mm})$ & Experimental & $\begin{array}{c}\text { Simulation } \\
(6 \mathrm{~dB} \text { bandwidth) }\end{array}$ & $\begin{array}{c}\text { Simulation } \\
\text { (single frequency) }\end{array}$ \\
\hline \multirow{2}{*}{ MSWQC45 } & 1.0 & -4.3 & -4.5 & -4.3 \\
& 1.5 & -1.8 & -1.8 & -2.0 \\
\hline \multirow{2}{*}{ WB45 } & 2.0 & 0 & 0 & 0 \\
& 1.0 & -2.6 & -3.9 & -1.0 \\
& 1.5 & -1.2 & -1.9 & 0 \\
\hline
\end{tabular}

In UTDefect the side-drilled hole is modeled by an infinitely long cylindrical cavity, and the exact solution obtained by separation of variables is used. Even if each hole has a finite length, it is reasonable to believe that the error introduced by considering a hole of infinite length is small.

In Table 1 the results from UTDefect are compared with the experimental results. The relative amplitudes in $\mathrm{dB}$ are given with the response from the hole of $2 \mathrm{~mm}$ diameter as reference value. As was mentioned previously, UTDefect works in the frequency domain, but results in the time domain can be obtained using a Fourier transform. In this specific case responses at about 60 different frequencies with a squared cosine spectrum, i.e. a Hanning window, have been used to determine the response. For comparison results obtained for a single frequency, the center frequency, have been included.

For probe MSWQC45 the agreement with the experimental results is excellent even for the case of a single frequency. For probe WB45 the agreement is not quite as good, but still well below $2 \mathrm{~dB}$, which must be considered as satisfactory. A possible explanation for the difference between the two probes is that for WB45 the defect is located in the near field of the probe, which means that the approximation by an infinite cylindrical cavity is not quite as good. It should also be noted that the probe is modeled by the traction exerted on the contact surface. Since the traction is unknown, this is a possible source of error.

\section{The Flat-Bottomed Hole}

Scattering from a flat-bottomed hole $(\mathrm{FBH})$ of diameter $3 \mathrm{~mm}$ oriented at $45^{\circ}$ relative to the normal of the scanning surface was studied. The center of the FBH was positioned at $30 \mathrm{~mm}$ depth. In UTDefect the flat-bottomed hole is modeled by a penny-shaped crack normal to the direction of incidence. This should be a good approximation as long as the diameter of the hole is not too small compared with the wavelength, as is the case in this benchmark problem. To solve the scattering problem an integral equation method originally developed by Krenk and Schmidt [1] is used. The basic idea is to derive an integral equation for the crack opening displacement (COD), and to solve the equation by expanding the COD in suitable functions that have the correct square root behavior at the edge of the crack. In UTDefect the T matrix of the penny-shaped crack is employed. A detailed derivation of the T matrix was given by Boström and Eriksson [2].

The results of the modeling are compared with the experimental results in Table 2, which gives the relative amplitudes in $\mathrm{dB}$ with the response from a side-drilled hole of diameter $2 \mathrm{~mm}$ as a reference. In this case 50-60 frequencies were used to obtain results in the time domain. Once again UTDefect gives results that differ by less than $2 \mathrm{~dB}$ from the 
TABLE 2. Response from a flat-bottomed hole.

\begin{tabular}{|c|c|c|c|}
\hline Probe & Experimental & $\begin{array}{c}\text { Simulation } \\
\text { (6 dB bandwidth) }\end{array}$ & $\begin{array}{c}\text { Simulation } \\
\text { (single frequency) }\end{array}$ \\
\hline $\begin{array}{c}\text { MSWQC45 } \\
\text { WB45 }\end{array}$ & 3.9 & 5.0 & 5.1 \\
\hline
\end{tabular}

experiments. It may be noted that for probe WB45 the results for a single frequency are in even better agreement than for the case of a full frequency spectrum.

\section{The Rectangular Crack}

Experimental results for scattering from rectangular surface-breaking cracks of different dimensions were also included in the benchmark problems. All cracks were normal to the back surface of the test block. The length of the crack, measured parallel to the back surface, was either 5 or $40 \mathrm{~mm}$, and the height varied from 2 to $20 \mathrm{~mm}$. In UTDefect the problem of scattering from rectangular and strip-like cracks is solved by a hypersingular integral equation method, see Jansson [3], and Bövik and Boström [4] for details. The solution that is implemented in UTDefect is only valid for non-surface breaking rectangular cracks, but as long as the wavelength is not too small compared to the dimensions of the crack it should be sufficient to consider a crack that is close to the back surface. For strip-like cracks, however, also surface-breaking cracks are modelled.

For the cracks of $5 \mathrm{~mm}$ length the comparison between experimental results and simulations are presented in Table 3 for the two different probes. In the calculations presented here, the distance between the edge of the crack and the back wall was taken as $0.1 \mathrm{~mm}$. The results in the time domain were obtained using about 50 frequencies for all cases. All results differ by less than $3 \mathrm{~dB}$ from the experimental values. It should be noted that for probe WB45 no results are given in the time domain for the largest crack because of memory allocation problems.

For the cracks of $40 \mathrm{~mm}$ length it is in most cases difficult to use the solution for scattering from a rectangular crack due to the excessive amount of memory needed. In many cases it should be possible to approximate the rectangular crack by a strip-like crack, i.e. a

TABLE 3. Response from rectangular cracks of length $5 \mathrm{~mm}$ and different heights.

\begin{tabular}{|c|c|c|c|c|}
\hline Probe & Height (mm) & Experimental & $\begin{array}{c}\text { Simulation } \\
\text { (6 dB bandwidth) }\end{array}$ & $\begin{array}{c}\text { Simulation } \\
\text { (single frequency) }\end{array}$ \\
\hline \multirow{3}{*}{ MSWQC45 } & 2 & 5.6 & 5.3 & 5.0 \\
& 5 & 9.0 & 9.8 & 10.1 \\
& 10 & 10.5 & 9.3 & 9.8 \\
WB45 & 20 & 11.3 & 9.1 & 9.1 \\
& 5 & 5.5 & 3.7 & 2.6 \\
& 10 & 11.1 & 9.6 & 8.3 \\
& 20 & 13.7 & 12.7 & 12.2 \\
\hline
\end{tabular}


TABLE 4. Response from rectangular cracks of length $40 \mathrm{~mm}$ and different heights.

\begin{tabular}{|c|c|c|c|c|}
\hline Probe & Height (mm) & Experimental & $\begin{array}{c}\text { Simulation } \\
\text { (6 dB bandwidth) }\end{array}$ & $\begin{array}{c}\text { Simulation } \\
\text { (single frequency) }\end{array}$ \\
\hline \multirow{2}{*}{ MSWQC45 } & 2 & 8.0 & 6.5 & 6.9 \\
& 5 & 13.3 & 13.4 & 13.4 \\
& 10 & 13.7 & 19.3 & 18.9 \\
WB45 & 20 & 13.5 & 16.4 & 16.3 \\
& 2 & 13.0 & 11.3 & 11.0 \\
& 10 & 18.1 & 15.5 & 14.3 \\
& 20 & 21.2 & 16.1 & 16.4 \\
\hline
\end{tabular}

crack of infinite length. In particular, this should work well for probe MSWQC45, where the defect is outside the near field of the probe. In Table 4 the results obtained for strip-like cracks of different heights are compared to the experimental results. To obtain the time traces 60-180 frequencies were used. As long as the height of the crack is not larger than $5 \mathrm{~mm}$ all results in the time domain are within $3 \mathrm{~dB}$ of the experimental values. For the larger cracks the agreement is not quite as good, in particular not for probe WB45, where the defect is inside the near field of the probe. A simple but possible explanation is that the strip-like crack is not a very good approximation for this case.

\section{SCATTERING FROM A RECTANGULAR CRACK IN A CLADDING}

In this section the most recent addition to UTDefect, scattering from a crack in a cladding, is presented.

\section{Background}

In the nuclear power industry thick plates or thick-walled pipes with an austenitic cladding for corrosion protection are used frequently. One way to apply the cladding is to use a welding process. This may lead to a couple of complications, when the component is subject to ultrasonic testing. The interface between the cladding and the base material is normally corrugated, which may cause unexpected scattering. Furthermore, the cladding material is anisotropic, which will also affect the wave propagation in the component.

A fully three-dimensional analytical model for a thick plate with a rectangular crack in a cladding has been developed taking the effects of a periodic interface as well as anisotropy into account. The problem is solved using a hypersingular integral equation approach. The solution is exact in the sense that no restrictions are imposed on the frequency or the shape of the periodic interface, although only a sinusoidal surface has been incorporated into UTDefect. The results are believed to be valid not only for a plate but also for a thick-walled pipe, as long as the radius of the pipe is sufficiently large compared to the wavelength. 


\section{Problem Formulation}

The geometry of the wave propagation problem is depicted in Fig. 1. A thick plate is composed of two layers of different generally anisotropic materials in welded contact. The crystal axes may be arbitrarily oriented. The interface $S_{0}$ is assumed to be periodic with a period $a$. This is believed to be a reasonable approximation, since real interfaces are more or less periodic. The layer of thickness $d_{1}$ is the base material. The cladding of thickness $d_{2}$ contains a rectangular crack $S_{\mathrm{C}}$, which may be tilted arbitrarily with respect to the back wall $S_{2}$. An ultrasonic transmitter is placed on the free surface $S_{1}$ of the base material. satisfy

For time-harmonic conditions the displacement fields $u_{j}^{i}$ in the two materials $i=1,2$

$$
\frac{\partial}{\partial x_{m}} \sigma_{m j}^{i}+\rho^{i} \omega^{2} u_{j}^{i}=0
$$

where $\omega$ is the angular frequency, $\rho^{i}$ is the density, and $\sigma_{m j}^{i}$ is the stress tensor, which is related to the displacement by the constitutive relation

$$
\sigma_{m j}^{i}=c_{m j m^{\prime} j^{\prime}}^{i} \frac{\partial}{\partial x_{j^{\prime}}} u_{m^{\prime}}^{i}
$$

The boundary conditions to be satisfied are $t_{j}=\sigma_{k j} n_{k}=0$ on $S_{1}$ (except directly below the transducer), $S_{2}$, and $S_{\mathrm{C}}$. Furthermore, $u_{i}$ and $t_{i}$ are continuous on the interface $S_{0}$, $x_{3}=s\left(x_{1}\right)$, which is assumed to be periodic. Actually, real interfaces are more or less sinusoidal. It should be pointed out, however, that there is no fundamental difficulty in choosing some other periodic function, as long as it is differentiable.

\section{Method of Solution}

The first step in the solution is to derive a Green's function for the same structure without a crack with a source in material 2. This Green's tensor can be used to derive an integral representation for the displacement field in the structure with a crack and with an incident field generated by an ultrasonic transmitter. From the integral representation it is straightforward to derive a hypersingular integral equation for the crack opening displacement (COD). Solution of the integral equation yields the COD, which can be used in Auld's reciprocity relation [5] to determine the change in signal response due to the crack. For a

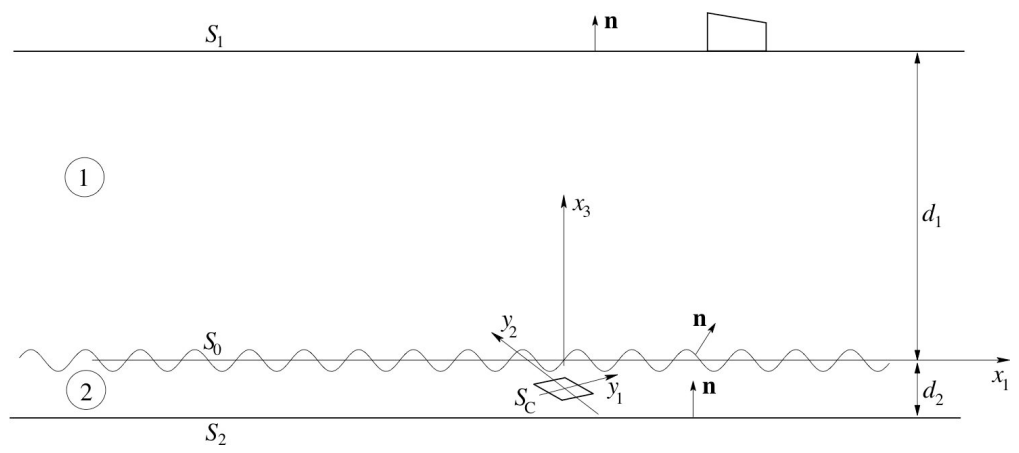

FIGURE 1. The geometry of a plate with a crack inside a cladding. 
more detailed description of the procedure the reader is referred to the corresponding twodimensional problem that was solved by Jansson and Zagbai [6].

\section{Numerical Results}

There are numerous parameters that can be varied in this problem. Here only some results showing the effect of the amplitude of the corrugated interface will be presented. In this example the base material is taken as an isotropic steel with density $\rho^{1}=8.4 \mathrm{~g} / \mathrm{cm}^{3}$, and and with logitudinal wave velocity $5.9 \mathrm{~mm} / \mu \mathrm{s}$ and shear velocity $3.2 \mathrm{~mm} / \mu \mathrm{s}$. The cladding material is assumed to be transversely isotropic with density $\rho^{2}=8.5 \mathrm{~g} / \mathrm{cm}^{3}$, and stiffness constants (in GPa) $c_{11}^{2}=216, c_{12}^{2}=c_{13}^{2}=115, c_{33}^{2}=250$, and $c_{44}^{2}=100$. The crystal axes coincide with the coordinate axes. The thicknesses of the base material and the cladding are $25 \mathrm{~mm}$ and $5 \mathrm{~mm}$, respectively. The crack is assumed to be quadratic, 2 by $2 \mathrm{~mm}$, and parallel to the scanning surface with its center $2.5 \mathrm{~mm}$ above the back surface at $x_{1}=0$. A 10 by $10 \mathrm{~mm}$ rectangular probe is transmitting longitudinal waves of fixed frequency $1 \mathrm{MHz}$ normal to the scanning surface. The interface is taken as sinusoidal with a period $a=5 \mathrm{~mm}$ and an amplitude $b$. In Fig. 2 the change in signal response caused by the crack is plotted against the position of the transducer for three different values of the amplitude $b$. It can be seen that an amplitude of $1 \mathrm{~mm}$ will cause a decrease in signal response of about $3 \mathrm{~dB}$ compared to the case of a flat interface.

\section{CONCLUDING REMARKS}

The experimental results for the 2009 ultrasonic benchmark problems from CEA have been compared to simulations obtained by the program UTDefect. With exception for a few cases with very large cracks the agreement is found to be quite satisfactory. UTDefect is a program that is developed continuously. A short description of the latest addition to the program, scattering from a crack in a cladding, has been presented with some preliminary results showing the effect of a corrugated boundary between the base material and the cladding.

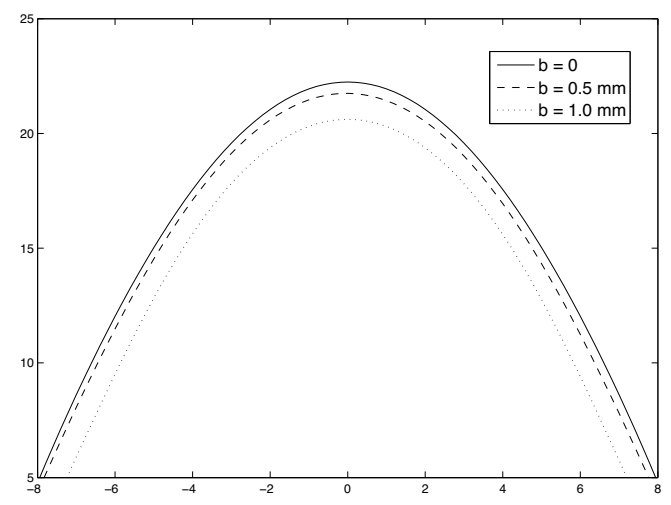

FIGURE 2. Signal response $(\mathrm{dB})$ vs position of the transducer $\left(x_{1}, \mathrm{~mm}\right)$ for different values of the amplitude (b) of the interface. 


\section{ACKNOWLEDGMENTS}

The present work is sponsored by the Swedish Radiation Safety Authority, and this is gratefully acknowledged.

\section{REFERENCES}

1. S. Krenk and H. Schmidt, Philos. Trans. R. Soc. London A 308, 167-198 (1982).

2. A. Boström and A. S. Eriksson, Proc. R. Soc. London A 443, 183-201 (1993).

3. P. Å. Jansson, "Modeling of ultrasonic detection of a rectangular crack," in Review of Progress in Quantitative Nondestructive Evaluation 19A, edited by D. O. Thompson and D. E. Chimenti, AIP Conference Proceedings vol. 509, American Institute of Physics, Melville, NY, 2000, pp. 57-63.

4. P. Bövik and A. Boström, J. Acoust. Soc. Am. 102, 2723-2733 (1997).

5. B. A. Auld, Wave Motion 1, 3-10 (1979).

6. P. Å. Jansson and T. Zagbai, "2D P-SV wave scattering by a crack in a cladding," in Review of Progress in Quantitative Nondestructive Evaluation 26A, edited by D. O. Thompson and D. E. Chimenti, AIP Conference Proceedings vol. 894, American Institute of Physics, Melville, NY, 2007, pp. 71-78. 